\title{
A Note on the Approximation Entropies of Certain Shifts
}

\author{
By
}

\author{
Kiichi NisHIUCHI*
}

\begin{abstract}
Voiculescu has proposed several routes to quantum entropies. Among them, the notion of the "approximation entropies" is a group of four entropies with similar definitions, based on two kinds of approximations. The $\mathrm{C}^{*}$-cases are extensions of the classical topological entropy and the $\mathrm{W}^{*}$-cases are those of the measure-theoretic one. In this paper, we will focus on the approximation entropies and investigate the entropies of Powers' binary shifts with some condition and the Jones shifts.
\end{abstract}

\section{§1. Preliminaries}

Voiculescu [15] has introduced quantum entropies of automorphisms of operator algebras, called approximation entropies. In this section, we will review the definitions and fix the notations. Using two kinds of approximation for the $\mathrm{W}^{*}$-case and the $\mathrm{C}^{*}$-case, Voiculescu has defined four approximation entropies which have similar definitions to each other. As Voiculescu said, one may think of approximation entropies as "growth"-entropies and the key concept is the " $\delta$-rank". The four approximation entropies are defined in the same way, as a matter of form, except for the " $\delta$-rank". So we will only state the definition by subalgebra approximation for the $\mathrm{W}^{*}$-case in detail. (see [15] for the other cases.)

Let $\mathscr{M}$ be a hyperfinite von Neuman algebra with a faithful normal tracial state $\tau$ and $\mathscr{F}(\mathscr{M})$ be the set of the unital finite-dimensional $\mathrm{C}^{*}$-subalgebras of $\mathscr{M}$. By $\mathscr{P} f(\mathscr{M})$ we denote the set of finite subsets of $\mathscr{M}$ and by $\operatorname{Aut}(\mathscr{M})$ the automorphism group of $\mathscr{M}$. For a normal faithful state $\varphi$ on $\mathscr{M}$, set $\operatorname{Aut}(\mathscr{M}, \varphi)=\{\alpha \in \operatorname{Aut}(\mathscr{M}) \mid \varphi \circ \alpha=\varphi\} . \quad$ For $\omega \in \mathscr{P} f(\mathscr{M})$ and $\mathscr{X} \subset \mathscr{M}$, we shall write

Communicated by Y. Takahashi, March 8, 1996.

1991 Mathematics Subject Classification(s): 46L55

* Department of Mathematical Sciences University of Tokyo, 3-8-1 Komaba Meguroku, Tokyo, Japan 
$\omega \subset_{\delta} \mathscr{X}$ if for every $a \in \omega$ there exists $x \in \mathscr{X}$ such that $\|a-x\|_{2}<\delta$ where $\|y\|_{2}=\tau\left(y^{*} y\right)^{1 / 2}$ for $y \in \mathscr{M}$. If $A \in \mathscr{F}(\mathscr{M})$ we denote by $r(A)$ its rank, i.e. the dimension of a maximal abelian self-adjoint subalgebra of $A$. For $\omega \in \mathscr{P} f(\mathscr{M})$ and $\delta>0$, we define the $\delta$-rank with respect to $\tau$ of $\omega$ as follows.

$$
r_{\tau}(\omega \mid \delta)=\inf \left\{r(B) \mid \omega \subset_{\delta} B \in \mathscr{F}(\mathscr{M})\right\} .
$$

For $\alpha \in \operatorname{Aut}(\mathscr{M}, \tau), \delta>0$ and $\omega \in \mathscr{P} f(\mathscr{M})$, we define

$$
\begin{aligned}
h a_{\tau}(\alpha, \omega \mid \delta) & =\limsup _{n \rightarrow \infty} \frac{1}{n} \log r_{\tau}\left(\omega \cup \alpha(\omega) \cup \cdots \cup \alpha^{n-1}(\omega) \mid \delta\right), \\
h a_{\tau}(\alpha, \omega) & =\sup _{\delta>0} h a_{\tau}(\alpha, \omega \mid \delta), \\
h a_{\tau}(\alpha) & =\sup \left\{h a_{\tau}(\alpha, \omega) \mid \omega \in \mathscr{P} f(\mathscr{M})\right\} .
\end{aligned}
$$

This $h a_{\tau}(\alpha)$ is called the approximation entropy of $\alpha$.

One can easily see that $r_{\tau}(\omega \mid \delta)$ is increasing in $\omega$ and decreasing in $\delta$ and $r_{\tau}(\alpha(\omega) \mid \delta)=r_{\tau}(\omega \mid \delta)$ for any $\alpha \in \operatorname{Aut}(\mathscr{M}, \tau)$.

In the $\mathrm{C}^{*}$-case, we replace hyperfinite von Neumann algebras by unital $\mathrm{AF}$-algebras and $c_{\delta}$ with respect to the 2-norm $\|\cdot\|_{2}$ by the $\mathrm{C}^{*}$-norm $\|\cdot\|$. Almost all the definitions are repetitions of the $\mathrm{W}^{*}$-case, so they are omitted. No state specified, we write $r(\omega \mid \delta)$, hat $(\alpha, \omega \mid \delta), \operatorname{hat}(\alpha, \omega)$ and $\operatorname{hat}(\alpha)$ for the $\mathrm{C}^{*}$-versions of $r_{\tau}(\cdot \mid \delta), h a_{\tau}(\alpha, \omega \mid \delta), h a_{\tau}(\alpha, \omega)$ and $h a_{\tau}(\alpha)$, respectively. We call hat $(\alpha)$ the topological approximation entropy of $\alpha$.

Now we define another $\delta$-rank by an approximation based on completely positive maps instead of subalgebras. Let $\mathscr{M}$ be a hyperfinite von Neumann algebra with a faithful normal state $\varphi$. By $C P A(\mathscr{M}, \varphi)$ we denote the set of triples $(\psi, \rho, B)$, where $B$ is a finite-dimensional $C^{*}$-algebra, $\psi: \mathscr{M} \rightarrow B$ and $\rho: B \rightarrow \mathscr{M}$ are unital completely positive maps such that $\varphi \circ \psi \circ \rho=\varphi$. For $\omega \in \mathscr{P} f(\mathscr{M})$ and $\delta>0$, the completely positive $\delta$-rank with respect to $\varphi$ of $\omega$ is defined as

$$
r_{\varphi}^{c p}(\omega \mid \delta)=\inf \left\{r(B) \mid(\psi, \rho, B) \in C P A(\mathscr{M}, \varphi),\|(\rho \circ \psi)(a)-a\|_{\varphi}<\delta \text { for } a \in \omega\right\},
$$

where $\|y\|_{\varphi}=\varphi\left(y^{*} y\right)^{1 / 2}$ for $y \in \mathscr{M}$. We can define $h a_{\varphi}^{c p}(\alpha, \omega \mid \delta), h a_{\varphi}^{c p}(\alpha, \omega)$ and $h a_{\varphi}^{c p}(\alpha)$ as in the subalgebra approximation, and $h a_{\varphi}^{c p}(\alpha)$ is called the completely positive approximation entropy of $\alpha$. In the $\mathrm{C}^{*}$-case of the completely positive map approximation, the norm $\|\cdot\|_{\varphi}$ is replaced by the $\mathrm{C}^{*}$-norm and $\mathscr{M}$ by a 
nuclear unital $C^{*}$-algebra with no state specified. As in the above cases, we can define $r^{c p}(\cdot \mid \delta), h t(\alpha, \omega \mid \delta), h t(\alpha, \omega)$ and $h t(\alpha)$ wich is called the topological entropy of $\alpha$.

Of cource, the approximation entropies are extensions of the classical entropy to the noncommutative framework (i.e. the $\mathrm{W}^{*}$-cases are those of the measure-theoretic Kolmogorov-Sinai entropy and the $\mathrm{C}^{*}$-cases are those of the topological entropy), that is, they coincide with classical ones in commutative cases. Furthermore, Voiculescu has shown that all the approximation entropies have the Kolmogorov-Sinai type theorem.

It is natural to ask what kinds of relations to other entropies they have. In general, one knows that Voiculescu's approximation entropies are larger than or equal to the Connes-Narnhofer-Thirring entropy (CNT-entropy, for short) and in special cases (for example, for noncommutative Bernoulli shifts), they are equal to each other. On the other hand, relations among approximation entropies are, roughly speaking, $h a_{\tau}^{c p} \leq h a_{\tau} \leq h a t \geq h t$. All the above comparisons were done by Voiculescu in [15].

\section{§2. Approximation Entropies of the Binary Shifts and the Jones Shifts}

In this section, we will estimate the approximation entropies of the binary shifts and the Jones shifts and determine their values.

\subsection{The binary shifts}

First, we will review the fundamental results of the binary shifts.

Let $(a)=\left\{a_{0}, a_{1}, \cdots\right\}$ be a sequence of 0 's and 1's with $a_{0}=0$. For such a bitstream $(a)$, we consider a sequence of hermitian unitary operators, $\left\{u_{i} \mid i \in Z\right\}$ satisfying the following "commutation relations"

$$
u_{j} u_{k}=(-1)^{a_{|j-k|}} u_{k} u_{j}
$$

Price [12] has shown that the von Neumann algebra $R$ generated by $\left\{u_{i} \mid i \in Z\right\}$ is isomorphic to the hyperfinite $\mathrm{II}_{1}$-factor if and only if the corresponding bitstream $(a)$ is not mirror-periodic (i.e. $\cdots, a_{2}, a_{1}, a_{0}, a_{1}, a_{2}, \cdots$ is not periodic.) From now we shall always assume that $(a)$ is a non-mirrorperiodic bitstream. Then, $R$ has the unique faithful normal tracial state $\tau$, which satisfies the following.

$$
\tau(w)=0, \quad \text { for any nontrivial word } w \text { of } u_{i} \text { 's. }
$$


Powers' binary shift is the unique extension of the mapping defined by $\sigma\left(u_{i}\right)=u_{i+1}$ to an automorphism on $R$, which we also denote by $\sigma$. And we call the extension on the $C^{*}$-algebra generated by $\left\{u_{i} \mid i \in \mathbb{Z}\right\}$ the topological binary shift. For computations of the entropy of binary shifts, we review the structure of the subalgebra of $R$ generated by $u_{0}, \cdots, u_{n}$, which we denote by $B_{n}$. We can easily see that $\operatorname{dim} B_{n}=2^{n+1}$. Furthermore, it is known $[11,13]$ that the dimension of the center of $B_{n}$ is $2^{c_{n}}$ for some $c_{n} \in\{0,1,2, \cdots\}$, and the algebra $B_{n}$ decomposes as the direct sum of $2^{c_{n}}$ copies of $2^{m_{n}} \times 2^{m_{n}}$ matrix algebras, where $m_{n}=(1 / 2)\left(n+1-c_{n}\right)$. We call the sequence $\left\{c_{0}, c_{1}, \cdots\right\}$ the center sequence for the shift corresponding to the bitstream (a). Powers and Price [11] determined the form of center sequences. The center sequence consists of a disjoint union of infinitely many finite strings of the form $12 \cdots m-1 m$ $m-1 \cdots 210$. The value of $m$ may vary in the sequence. Furthermore, they have shown in [11] that if a bitstream $(a)$ is eventually periodic, that is, for some $p \in N$ the subsequence $a_{p} a_{p+1} \cdots$ of $(a)$ is periodic, then the center sequence associated with $(a)$ is also eventually periodic.

Now, we have prepared for calculation of the entropy of the binary shifts. By using the tensor product inequality and the monotonicity of $h a_{\tau}^{c p}$, Voiculescu has shown in [15] that for any Powers' binary shift $\sigma$,

$$
\frac{1}{2} \log 2 \leq h a_{\tau}^{c p}(\sigma) \leq h a_{\tau}(\sigma) \leq \log 2 .
$$

Similarly to the above result, for any topological binary shift $\sigma$, we have

$$
\frac{1}{2} \log 2 \leq h t(\sigma) \leq h a t(\sigma) \leq \log 2 .
$$

Proposition 2.1. Let $\sigma$ be the Powers' binary shift automorphism with the corresponding bitstream eventually periodic. Then, all the approximation entropies are $(1 / 2) \log 2$.

Proof. By virtue of the Voiculescu's result, it suffices to show that $h a t(\sigma) \leq(1 / 2) \log 2$, since $h a_{\tau}(\sigma) \leq h a t(\sigma)$ by Proposition 2.4. in [15].

Let $B_{n}$ be the algebra generated by $\left\{u_{0}, u_{1}, \cdots, u_{n}\right\}$ and $c_{n}$ be its center sequence. Then, $B_{n}$ is the direct sum of $2^{c_{n}}$ copies of $2^{m_{n}} \times 2^{m_{n}}$ matrix algebras, where $m_{n}=(1 / 2)\left(n+1-c_{n}\right)$. Hence, $r\left(B_{n}\right)=2^{(1 / 2)\left(n+1+c_{n}\right)}$. Since $r\left(\left\{u_{0}, u_{1}, \cdots\right.\right.$, $\left.\left.u_{n}\right\} \mid \delta\right) \leq r\left(B_{n}\right)$ for any $\delta>0$, 


$$
\begin{aligned}
\operatorname{hat}\left(\sigma, u_{0}\right) & \leq \limsup _{n} \frac{1}{n+1} \log r\left(B_{n}\right) \\
& =\limsup _{n} \frac{n+1+c_{n}}{2(n+1)} \log 2 .
\end{aligned}
$$

Since $\left\{c_{n}\right\}$ is eventually periodic, $\left\{c_{n}\right\}$ is bounded, and by Proposition 2.3. in [15], we get

$$
\operatorname{hat}(\sigma)=\operatorname{hat}\left(\sigma, u_{0}\right) \leq \frac{1}{2} \log 2
$$

\subsection{The Jones shifts}

Let $\left\{e_{i} \mid i \in Z\right\}$ be a sequence of projections satisfying the following "Temperly-Lieb relation".

(1) $e_{i} e_{j}=e_{j} e_{i}$, for $|i-j| \geq 2$,

(2) $e_{i} e_{i \pm 1} e_{i}=\lambda e_{i}$,

where $\lambda \in\left(0, \frac{1}{4}\right] \cup\left\{\left(4 \cos ^{2} \frac{\pi}{m}\right)^{-1} \mid m \in N, m \geq 3\right\}$.

As shown by Jones [8], the von Neumann algebra generated by $\left\{e_{i} \mid i \in Z\right\}$ and the unit 1 , which we denote by $R$, is the hyperfinite $\mathrm{II}_{1}$-factor with the canonical tracial state $\tau$. The Jones shift is the automorphism on $R$ defined by $\theta_{\lambda}\left(e_{i}\right)=e_{i+1}$. We will also use the same notation when we consider the Jones shifts on the $\mathrm{C}^{*}$-algebra generated by $\left\{e_{i} \mid i \in Z\right\}$.

Various entropies of the Jones shifts have been already calculated, and we will recall some of the results here. (In connection with the index theory for type $\mathrm{II}_{1}$ subfactors, one can find deeper results on the entropies in [3], [4] and [6], viewing the Jones shifts as the square roots of the canonical shifts.)

Pimsner and Popa [10] computed the Connes-Størmer entropy of the Jones shifts, except for the case $\lambda=1 / 4$ which was settled by Yin [16] and Choda [2]. One who wants to know the whole treatment of the CNT-version of the calculation may refer to Section 17 in [9]. It is known that

$$
h_{\tau}\left(\theta_{\lambda}\right)= \begin{cases}-\frac{1}{2} \log \lambda, & \text { when } \frac{1}{4}<\lambda \leq 1 \\ \eta(t)+\eta(1-t), & \text { when } 0<\lambda \leq \frac{1}{4}, \quad \text { where } t(1-t)=\lambda\end{cases}
$$


where $h_{\tau}(\cdot)$ is the CNT-entropy with respect to $\tau$ and $\eta(t)=-t \log t$.

Let $A_{n}$ be the $\mathrm{C}^{*}$-subalgebra of $R$ generated by $1, e_{0}, \cdots, e_{n}$ and $\mathscr{A}_{l o c}=\cup_{n} A_{n}$, which is a local $\mathrm{C}^{*}$-algebra with $\theta_{\lambda}\left(\mathscr{A}_{\text {loc }}\right) \subset \mathscr{A}_{\text {loc }}$. (In this case, we think of $\theta_{\lambda}$ as a unital $*$-endomorphism on $\mathscr{A}_{\text {loc }}$ ). It is known [14] that the Thomsen's topological entropy $\hbar$ of the Jones sifts has the following formula and one can compute the value with it.

$$
\hbar\left(\left.\theta_{\lambda}\right|_{\mathscr{A}_{l o c}}\right)=\quad \limsup _{n} \frac{\log r\left(A_{n}\right)}{n}= \begin{cases}-\frac{1}{2} \log \lambda, & \text { when } \frac{1}{4}<\lambda \leq 1, \\ \log 2, & \text { when } 0<\lambda \leq \frac{1}{4} .\end{cases}
$$

Of cource, we can think of $\theta_{\lambda}$ as an automorphism for which there is a canonical local C*-subalgebra $A$ of $R$ such that $\theta_{\lambda}(A)=A$. For this automorphic version, we obtain exactly the same result as above.

Proposition 2.2. If $\frac{1}{4} \leq \lambda<1$, then all the approximation entropies are $-\frac{1}{2} \log \lambda$

Proof. By Propositions 3.6, 3.7, 2.4, 4.6 and 4.5. in [15], we have $h_{\tau}\left(\theta_{\lambda}\right) \leq h a_{\tau}^{c p}\left(\theta_{\lambda}\right) \leq h a_{\tau}\left(\theta_{\lambda}\right) \leq \operatorname{hat}\left(\theta_{\lambda}\right)$ and $h_{\tau}\left(\theta_{\lambda}\right) \leq h t\left(\theta_{\lambda}\right) \leq \operatorname{hat}\left(\theta_{\lambda}\right)$. Since $h_{\tau}\left(\theta_{\lambda}\right)=-\frac{1}{2} \log \lambda$, it is sufficient to show that $\operatorname{hat}\left(\theta_{\lambda}\right) \leq-\frac{1}{2} \log \lambda$.

Let $A_{n}$ be the finite-dimensional $C^{*}$-subalgebra generated by $\left\{e_{i} \mid 0 \leq i\right.$ $\leq n-1\}$. For any $\delta>0$, we have $r\left(\left\{e_{0}, \cdots, e_{n-1}\right\} \mid \delta\right) \leq r\left(A_{n}\right)$. By Proposition 2.3. in $[15]$,

$$
\operatorname{hat}\left(\theta_{\lambda}\right)=\operatorname{hat}\left(\theta_{\lambda}, e_{0}\right) \leq \limsup _{n} \log \frac{r\left(A_{n}\right)}{n}=\hbar\left(\theta_{\lambda}\right)=-\frac{1}{2} \log \lambda
$$

Remark. Here we return to Powers' binary shifts and consider the Powers' binary shift $\sigma_{0}$ corresponding to a bitstream $0100 \ldots$. This shift is very important, for all the Powers' binary shifts with commutant index 2 are cocycle conjugate to it, though there are countably many non-conjugate binary shifts 
of commutant index 2 (see [13]). And one knows that $\sigma_{0}$ is the Jones shift with $\lambda=1 / 2$, which is easily seen as follows.

Let $\left\{u_{i} \mid i \in Z\right\}$ be the hermitian unitary generators with respect to $\sigma_{0}$. Putting $e_{i}=(1 / 2)\left(1+u_{i}\right)$, one can easily see that $e_{i}$ 's are projections and satisfy the Temperly-Lieb relation with $\lambda=1 / 2$ and that $\sigma_{0}\left(e_{i}\right)=e_{i+1}$. Furthermore, of cource, the approximation entropies of $\sigma_{0}$ coincide with those of $\theta_{1 / 2}$ by Propositions 2.1 and 2.2.

Proposition 2.3. If $0<\lambda \leq \frac{1}{4}$, then $\operatorname{hat}\left(\theta_{\lambda}\right)=h t\left(\theta_{\lambda}\right)=\log 2$.

Proof. In this case, according to Jones' result [8], the $\mathrm{C}^{*}$-algebra generated by $\left\{e_{i} \mid i \in Z\right\}$ has the Bratteli diagram arising from reflections of Dynkin diagram $A_{\infty}$. As pointed out in Example 1.3 of [7], $\theta_{\lambda}$ are independent of $\lambda$ up to unitary equivalence. Hence, $h a t\left(\theta_{\lambda}\right)$ and $h t\left(\theta_{\lambda}\right)$ are also independent of $\lambda$. As in the previous proposition, we have

$$
h_{\tau}\left(\theta_{\lambda}\right) \leq h t\left(\theta_{\lambda}\right) \leq h a t\left(\theta_{\lambda}\right) \leq \hbar\left(\theta_{\lambda}\right)=\log 2, \quad \text { for all } \lambda \in(0,1 / 4] .
$$

Since $h_{\tau}\left(\theta_{\lambda}\right)=\eta(t)+\eta(1-t)$, where $t(1-t)=\lambda \leq 1 / 4, h_{\tau}\left(\theta_{\lambda}\right)$ is maximized at $\lambda=1 / 4$ and its maxium is $\log 2$. So we get this proposition.

Proposition 2.4. If $0<\lambda \leq \frac{1}{4}$, then $h a_{\tau}^{c p}\left(\theta_{\lambda}\right)=\eta(t)+\eta(1-t)$, where $t=$ $\frac{1+\sqrt{1-4 \lambda}}{2}$

Proof. Pimsner and Popa [10] have shown that in the case of $0<\lambda \leq \frac{1}{4}$, the Jones shift is a kind of the Bernoulli shift. To illustrate it, we consider the infinite tensor product of replicas of $2 \times 2$ matrix algebra $M_{2}(C)$. Let $M_{i}=M_{2}(C), M=\otimes_{i \in \mathbf{Z}} M_{i}$ and $M_{[n, m]}=\otimes_{i=n}^{m} M_{i}$. We denote the matrix units in $M_{n}$ by $w_{i j}^{(n)}(n \in Z, 1 \leq i, j \leq 2)$. Let $\varphi_{i}$ be a state with its density $t w_{11}^{(i)}+(1-t) w_{22}^{(i)}$ in $M_{i}$ and $\varphi$ be the product state $\otimes_{i \in Z} \varphi_{i}$ on $M$. For $n \in N$, we set

$$
\begin{aligned}
e_{n}= & \cdots 1 \otimes\left((1-t) w_{11}^{(n)} \otimes w_{22}^{(n+1)}+\sqrt{t(1-t)} w_{12}^{(n)} \otimes w_{21}^{(n+1)}\right. \\
& \left.+\sqrt{t(1-t)} w_{21}^{(n)} \otimes w_{12}^{(n+1)}+t w_{22}^{(n+1)} \otimes w_{11}^{(n+1)}\right) \otimes 1 \cdots
\end{aligned}
$$




$$
=\cdots 1 \otimes\left(\begin{array}{cccc}
0 & 0 & 0 & 0 \\
0 & 1-t & \sqrt{t(1-t)} & 0 \\
0 & \sqrt{t(1-t)} & t & 0 \\
0 & 0 & 0 & 0
\end{array}\right) \otimes 1 \cdots \quad \in M_{[n, n+1]} .
$$

It is straightforward to see that $e_{n}$ 's are projections and satisfy the Temperley-Lieb relation. So we can realize the Jones shifts with $\lambda<1 / 4$ on the infinite tensor product. Let $R$ be the AF-algebra generated by $\cdots, e_{-1}, e_{0}, e_{1}, \cdots$ and $\bar{\varphi}$ be the extension of $\varphi$ to $\pi_{\varphi}(M)^{\prime \prime}$, where $\pi_{\varphi}$ is the GNS-representation with respect to $\varphi$. We write $\gamma$ for the right shift on $M$ and $\bar{\gamma}$ for its extension on $\pi_{\varphi}(M)^{\prime \prime}$. Pimsner and Popa have shown that $\pi_{\varphi}(R)^{\prime \prime}$ is just the centralizer $\mathscr{Z}$ of $\bar{\varphi}$ and $\left.\bar{\gamma}\right|_{\mathscr{Z}}$ is the Jones shift. (It is also well-known that Connes and Størmer [5] have proved that $\mathscr{Z}$ is the hyperfinite $\mathrm{II}_{1}$ factor with the canonical tracial state $\left.\bar{\varphi}\right|_{\mathscr{Z}}$ and $H\left(\left.\bar{\gamma}\right|_{\mathscr{Z}}\right)=\eta(t)+\eta(1-t)$. They called this shift the Bernoulli shift defined by $\{1-t, t\}$.) Applying this idea to the approximation entropy, we get

$$
h_{\tau}\left(\theta_{\lambda}\right) \leq h a_{\tau}^{c p}\left(\theta_{\lambda}\right)=h a_{\bar{\varphi} \mid \mathscr{Z}}^{c p}\left(\left.\bar{\gamma}\right|_{\mathscr{Z}}\right) \leq h a_{\bar{\varphi}}^{c p}(\bar{\gamma})=h_{\bar{\varphi}}(\bar{\gamma}) .
$$

The first inequality and the second one are due to Proposition 3.6. and Proposition 3.5. in [15] respectively and the last equality is due to Proposition 3.9. in [15]. One knows that $h_{\tau}\left(\theta_{\lambda}\right)=h_{\bar{\varphi}}(\bar{\gamma})=\eta(t)+\eta(1-t)$. So we get this proposition.

Remark. By the same method as in Proposition 2.2, we can calculate the approximation entropies of other shifts. Let $S$ be a finite subset of $N$ and $n \in N$. Then there exists a family $\left\{u_{i} \mid i \in Z\right\}$ of unitaries satisfying the following conditions.

(1) $u_{i}^{n}=1, \quad$ for any $i \in \mathbb{Z}$,

(2) $u_{i} u_{j}=\exp (2 \pi \sqrt{-1} / n) u_{j} u_{i}, \quad$ if $|i-j| \in S$,

(3) $u_{i} u_{j}=u_{j} u_{i}, \quad$ if $|i-j| \notin S$.

Let $P$ be the von Neumann algebra generated by the family and $\theta$ be the automorphism defined by $\theta\left(u_{i}\right)=u_{i+1}$ on $P$. (We also write $\theta$ for the automorphism given by $u_{i} \rightarrow u_{i+1}$ on the $\mathrm{C}^{*}$-algebra generated by the family.) One knows that $P$ is the hyperfinite $\mathrm{II}_{1}$-factor and $h_{\tau}(\theta)=\hbar(\theta)$ $=(1 / 2) \log n$. (See [1], [2] and [14].) Since $\left\{u_{0}, \cdots, u_{k-1}\right\}$ generates a finite-dimensional $C^{*}$-subalgebra of $P$, as in Proposition 2.2, we obtain 


$$
h_{\tau}(\theta) \leq h a_{\tau}^{c p}(\theta) \leq h a_{\tau}(\theta) \leq h a t(\theta) \leq \hbar(\theta) \text { and } h_{\tau}(\theta) \leq h t(\theta) \leq h a t(\theta) \leq \hbar(\theta) .
$$

Hence, all the approximation entropies are $\frac{1}{2} \log n$.

\section{Acknowledgements}

The author thanks Prof. M. Choda for giving him the theme of this paper and much advice. And he is greatly indebted to Prof. F. Hiai for many helpful discussions. Finally, the author is very grateful to Prof. Y. Kawahigashi for answering his many questions, correcting his many mistakes and guiding him to oprator algebras.

\section{References}

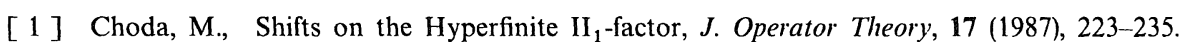

[ 2 ] Entropy for *-Endomorphisms and Relative Entropy for Subalgebras, J. Operator Theory, 25 (1991), 125-140.

[3] - Entropy for Canonical Shifts, Trans. Amer. Math. Soc., 334 (1992), 827-849.

[ 4 ] Choda, M. and Hiai, F., Entropy for Canonical Shifts. II, Publ. RIMS, Kyoto Univ., 27 (1991), 461-489.

[ 5 ] Connes, A. and Størmer, E., Entropy of $\mathrm{II}_{1}$ von Neumann algebras, Acta Math., 134 (1975), 289-306.

[6] Hiai, F., Entropy for Canonical Shifts and Strong Amenability, Internat. J. Math., 6 (1995), 381-396.

[ 7 ] Hiai, F. and Petz, D., Quantum Mechanics in AF C*-systems, Preprint.

[ 8 ] Jones, V. F. R., Index for Subfactors, Invent. Math., 72 (1983), 1-25.

[ 9 ] Ohya, M. and Petz, D., Quantum Entropy and Its Use, Berlin, Heidelberg, New York: Springer, 1993.

[10] Pimsner, M. and Popa, S.. Entropy and Index for Subfactors, Ann. Sci. École Norm. Sup. Sér.4, 19 (1986), 57-106.

[11] Powers, R. T. and Price, G. L., Cocycle Conjugacy Classes of Shifts on the Hyperfinite $\mathrm{II}_{1}$ Factor, J. Func. Anal., 121 (1994), 275-295.

[12] Price, G. L., Shifts on Type II, Factors, Canad. J. Math, 39 (1987), 492-511.

[13] - Cocycle Conjugacy Classes of Shifts on the Hyperfinite II Factor, II, Preprint.

[14] Thomsen, K., Topological Entropy for Endomorphisms of Local C*-Algebras, Comm. Math. Phys., 164 (1994), 181-193.

[15] Voiculescu, D., Dynamical Approximation Entropies and Topological Entropy in Operator Algebras, Comm. Math. Phys., 170 (1995), 249-281.

[16] Yin, H. S., Entropy of Certain Noncommutative Shifts, Rocky Mount. J. Math., 20 (1990), 651-656. 
